\title{
On the Relative Distances of Six Points in a Plane Convex Body
}

\author{
K. Böröczky and Z. Lángi
}

\begin{abstract}
Let $C$ be a convex body in the Euclidean plane. By the relative distance of points $p$ and $q$ we mean the ratio of the Euclidean distance of $p$ and $q$ to the half of the Euclidean length of a longest chord of $C$ parallel to $p q$. In this note we find the least upper bound of the minimum pairwise relative distance of six points in a plane convex body.
\end{abstract}

Denote the closed segment with endpoints $p$ and $q$ in the Euclidean plane $E^{2}$ by $p q$, and denote the Euclidean length of $p q$ by $|p q|$. Let $C \subset E^{2}$ be a convex body. Take a chord $p^{\prime} q^{\prime}$ of $C$ parallel to $p q$ such that there is no longer chord of $C$ parallel to $p q$. The ratio $d_{C}(p, q)$ of $|p q|$ to $\frac{1}{2}\left|p^{\prime} q^{\prime}\right|$ is called the $C$-distance of points $p$ and $q$ (see [5]). We also use the term $C$-length of the segment $p q$. If there is no doubt about $C$, we talk about the relative distance of points $p$ and $q$, or the relative length of the segment $p q$. If $C$ is centrally symmetric, the relative distance is the Minkowski distance in the Minkowski space whose unit ball is $C$.

It is shown by Doyle, Lagarias and Randall [2] that among six arbitrary points of an arbitrary centrally symmetric plane convex body there is a pair in relative distance at most 1 . They also showed that this value is attained for every centrally symmetric convex body. For some smaller values of $k$, they found a connection between the minimal pairwise relative distance of $k$ points in a centrally symmetric plane convex body on one hand, and the homothety ratio of $k$ congruent homothetical copies of the body packed into the body on the other hand.

The paper [1] of Doliwka and Lassak implies that among five arbitrary boundary points of an arbitrary plane convex body there exists a pair in relative distance at most $\sqrt{5}-1 \approx 1.236$. In the Appendix of our paper we show that the statement remains true if we consider arbitrary points (not obligatorily boundary points) of a convex body. The examples of the regular pentagon and the regular decagon show that the value $\sqrt{5}-1$ cannot be lessened.

Lángi [3] proved that among six arbitrary boundary points of an arbitrary plane convex body there exists a pair in relative distance at most $8-4 \sqrt{3} \approx 1.072$. This value is attained for the hexagon that is the convex hull of the regular triangle and its homothetical copy with center at the center of gravity of the triangle and with homothety ratio $1-\sqrt{3}$ (see [1] or [3]). This hexagon is nothing else but the convex hull of the vertices and of the midpoints of the arcs of the Reuleaux triangle. In this paper we prove the following theorem.

2000 Mathematics Subject Classification: 52A10, 52A40, 52C15

Key words and phrases: convex body, relative distance, homothety, packing Research supported by the Hungarian Scientific Foundation grant No. T037752 
Theorem. Among six arbitrary points of an arbitrary plane convex body there exists a pair in relative distance at most $2-\frac{2 \sqrt{5}}{5} \approx 1.106$. Furthermore, if $p_{1}, \ldots p_{6}$ are points in a plane convex body $C$ such that all their pairwise relative distances are at least $2-\frac{2 \sqrt{5}}{5}$, then $C$ is an affine regular pentagon and the points are its vertices and its center.

Using the idea of [1] and [5] we can immediately reformulate our Theorem in the following form (see also Theorem of [4]).

No plane convex body can be packed by its six homothetical copies of ratio greater than $\frac{9}{19}-\frac{1}{19} \sqrt{5}$. Moreover, if a plane convex body $C$ can be packed by its six homothetical copies of ratio $\frac{9}{19}-\frac{1}{19} \sqrt{5}$, then $C$ is an affine regular pentagon and the homothety centers are its vertices and its center.

During the proof of our theorem, we denote points by small Latin letters. In a Cartesian coordinate system, the $x$-coordinate and the $y$-coordinate of a point $p \in E^{2}$ are denoted by $p^{x}$ and $p^{y}$, respectively. We denote the straight line through the points $p, q \in E^{2}$ by $L(p, q)$. The value $2-\frac{2 \sqrt{5}}{5}$ is denoted by $\lambda$, the value $\frac{\lambda}{2}=1-\frac{\sqrt{5}}{5} \approx 0.553$ by $\tau$ and the value $\frac{\lambda}{2-\lambda}=\sqrt{5}-1$ by $\nu$. By the kernel of a convex pentagon $P$ we mean the convex pentagon which is bounded by the diagonals of $P$.

The proof of our theorem is based on three lemmas.

Lemma 1. Take a convex pentagon $P=a_{1} a_{2} a_{3} a_{4} a_{5}$ and take a point $p$ in the kernel of $P$. Denote $\min \left\{d_{P}\left(p, a_{i}\right) \mid i=1, \ldots, 5\right\}$ by $\lambda(P, p)$. Then $\lambda(P, p) \leq \lambda$ and equality holds if and only if $P$ is an affine regular pentagon and $p$ is its center.

Proof. Compactness arguments show that the maximal value of $\lambda(P, p)$ is attained on the family of convex pentagons $P$ and points $p$ of the kernel of $P$. Moreover, if $P$ is an affine regular pentagon and if $p$ is its center, then $\lambda(P, p)$ is equal to $\lambda$. Hence it is enough to show that if $P$ is not affine regular or if $p$ is not its center, then $\lambda(P, p)$ cannot be maximal. During the proof we denote the intersection point of the line $L\left(p, a_{i}\right)$ and of the opposite side of $P$ by $b_{i}$ for $i=1, \ldots, 5$. Moreover, we denote the kernel of $P$ by $Q$.

Observe that if $p$ is a point of the boundary of $Q$, then $\lambda(P, p) \leq 1$, which is less than $\lambda$. Thus in this case $\lambda(P, p)$ cannot be maximal. Therefore in the sequel we assume that $p$ is in the interior of $Q$.

Case 1, when $P$ has a side of $P$-length 2 . For instance, let $a_{1} a_{2}$ be such a side. Instead of the condition that $p$ is in the kernel of $P$, during the proof in this case we use only the terms that $p \in a_{1} a_{3} a_{5}$ and $p \in a_{2} a_{3} a_{5}$. For $i=1, \ldots, 5$ let us denote a maximal chord of $P$ parallel to $a_{i} p$ by $u_{i} v_{i}$. As $\left|u_{i} v_{i}\right| \geq\left|a_{i} b_{i}\right|$, we have $d_{P}\left(a_{i}, p\right)=\frac{\left|a_{i} p\right|}{\frac{1}{2}\left|u_{i} v_{i}\right|} \leq \frac{2\left|a_{i} p\right|}{\left|a_{i} b_{i}\right|}$.

If $\left|a_{5} p\right|>\left|p b_{5}\right|$ and if $\left|a_{3} p\right|>\left|p b_{3}\right|$, then $L\left(a_{1}, a_{2}\right)$ separates $p$ and the intersection point of $L\left(a_{1}, a_{5}\right)$ and $L\left(a_{2}, a_{3}\right)$. Thus $d_{P}\left(a_{1}, a_{2}\right)<2$, which is a contradiction. If $\left|a_{3} p\right| \leq\left|p b_{3}\right|$, then $d_{P}\left(a_{3}, p\right) \leq 1$, hence $\lambda(P, p) \leq 1$. Similarly, if $\left|a_{5} p\right| \leq\left|p b_{5}\right|$, then $\lambda(P, p) \leq d_{P}\left(a_{5}, p\right) \leq$ 1. 
Case 2, when $P$ has no side of $P$-length 2. In this case $d_{P}\left(p, a_{i}\right)=\frac{2\left|a_{i} p\right|}{\left|a_{i} b_{i}\right|}$ for $i=1, \ldots, 5$.

Subcase 2.1, when $P$ has two consecutive vertices in $P$-distance from $p$ greater than $\lambda(P, p)$. Assume, for example, that $d_{P}\left(a_{4}, p\right)>\lambda(P, p)$ and that $d_{P}\left(a_{5}, p\right)>\lambda(P, p)$ (see Figure 1$)$. For $i=1, \ldots, 5$ let us denote by $H_{i}$ the open halfplane bounded by the line through $p$ parallel to $a_{i+2} a_{i+3}$ such that $a_{i} \notin H_{i}$. Observe that if $p^{\prime}$ is in $H_{i}$, then $d_{P}\left(a_{i}, p\right)<d_{P}\left(a_{i}, p^{\prime}\right)$. Let $H$ be $H_{1} \cap H_{2} \cap H_{3}$. Notice that $H^{\prime}=H \cap$ int $Q$ is a nonempty open set, and that $p$ is a boundary point of $H^{\prime}$. If $p^{\prime}$ is a point of $H^{\prime}$, then $d_{P}\left(a_{i}, p^{\prime}\right)>d_{P}\left(a_{i}, p\right) \geq \lambda(P, p)$ for $i=1,2,3$. Moreover, if $p^{\prime}$ is close enough to $p$, then $d_{P}\left(a_{j}, p^{\prime}\right)>\lambda(P, p)$ for $j=4,5$. Thus, we can choose a point $p^{\prime} \in \operatorname{int} Q$ such that $\lambda(P, p)<\lambda\left(P, p^{\prime}\right)$. Hence $\lambda(P, p)$ cannot be maximal.

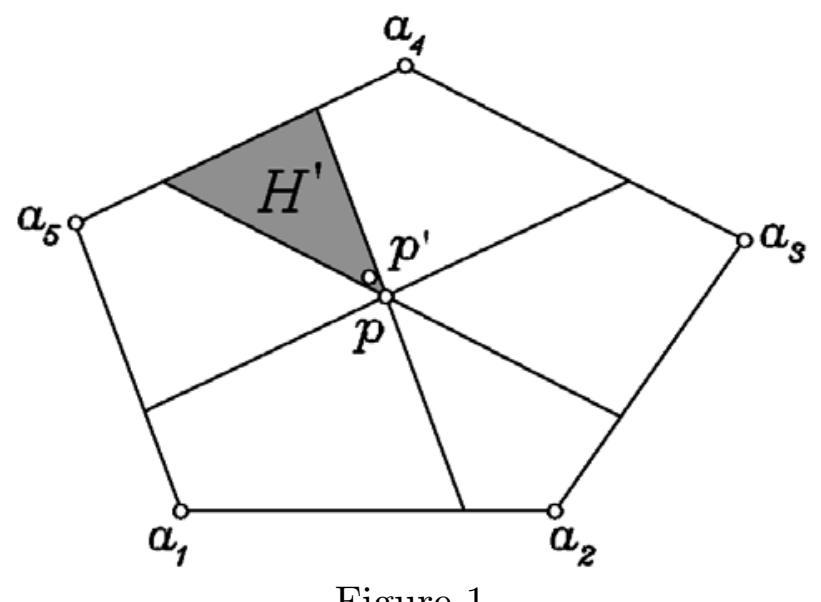

Figure 1

Subcase 2.2, when $P$ has exactly two, nonconsecutive vertices in $P$-distance from $p$ greater than $\lambda(P, p)$. Without loss of generality, let $d_{P}\left(a_{1}, p\right)=d_{P}\left(a_{2}, p\right)=d_{P}\left(a_{4}, p\right)=$ $\lambda(P, p), d_{P}\left(a_{3}, p\right)>\lambda(P, p)$ and $d_{P}\left(a_{5}, p\right)>\lambda(P, p)$. Take the convex pentagon $P^{\prime}=$ $a_{1} a_{2} a_{3} a_{4} a_{5}^{\prime}$, where $a_{5}^{\prime}$ is an interior point of the segment $a_{5} a_{1}$. We have $d_{P^{\prime}}\left(a_{2}, p\right)>$ $d_{P}\left(a_{2}, p\right)=\lambda(P, p)$ and $d_{P^{\prime}}\left(a_{5}^{\prime}, p\right)<d_{P}\left(a_{5}, p\right)$. Moreover, if $a_{5}^{\prime}$ is close enough to $a_{5}$, then $d_{P^{\prime}}\left(a_{5}^{\prime}, p\right)>\lambda(P, p)$, and $p$ is in the kernel of $P^{\prime}$. Hence, according to Subcase 2.1, there exists a point $p^{\prime}$ in the kernel of $P^{\prime}$ such that $\lambda(P, p)=\lambda\left(P^{\prime}, p\right)<\lambda\left(P^{\prime}, p^{\prime}\right)$.

Subcase 2.3, when $P$ has exactly one vertex in $P$-distance from $p$ greater than $\lambda(P, p)$. Let this vertex be $a_{5}$. Take the convex pentagon $P^{*}=a_{1} a_{2} a_{3} a_{4} a_{5}^{*}$, where $a_{5}^{*}$ is an interior point of the segment $a_{5} a_{1}$. We have that $d_{P^{*}}\left(a_{2}, p\right)>d_{P}\left(a_{2}, p\right)=\lambda(P, p)$ and $d_{P^{*}}\left(a_{5}^{*}, p\right)<$ $d_{P}\left(a_{5}, p\right)$. Moreover, if $a_{5}^{*}$ is close enough to $a_{5}$, then $d_{P^{*}}\left(a_{5}^{*}, p\right)>\lambda(P, p)$. Hence, thanks to Subcase 2.2, there exist a convex pentagon $P^{\prime}$ and a point $p^{\prime}$ in the kernel of $P^{\prime}$ such that $\lambda(P, p)=\lambda\left(P^{*}, p\right)<\lambda\left(P^{\prime}, p^{\prime}\right)$.

Subcase 2.4, when $d_{P}\left(a_{i}, p\right)=\lambda(P, p)$ for $i=1, \ldots, 5$. As we are looking for the maximal value of $\lambda(P, p)$, we assume that $\lambda(P, p)>1$. For the sake of simplicity, we use the notation $\nu(P, p)=\frac{\lambda(P, p)}{2-\lambda(P, p)}$. Thus $\frac{\left|a_{i} p\right|}{\left|p b_{i}\right|}=\nu(P, p)$ for $i=1, \ldots, 5$. Observe that $\nu(P, p)$ is a strictly increasing function of $\lambda(P, p)$. Additionally, $\lambda(P, p)>1$ implies that $\nu(P, p)>1$. Let $h_{p}$ be the homothety with homothety center $p$ and with homothety ratio 
$-\frac{1}{\nu(P, p)}$. Then $h_{p}\left(a_{i}\right)=b_{i}$ for $i=1, \ldots, 5$.

Consider the intersection point $a$ of the lines $L\left(a_{1}, a_{5}\right)$ and $L\left(a_{2}, a_{3}\right)$. Let us take a Cartesian coordinate system. As the relative distance of two points does not change under affine transformations, we can assume that the points $a, a_{1}, a_{2}$ are $(0,0),(1,-1),(-1,-1)$, respectively (see Figure 2).

We intend to show that if $p^{x} \neq 0$, then $\lambda(P, p)$ is not maximal. Assume that $p^{x}>0$ (in the other case the proof is analogous).

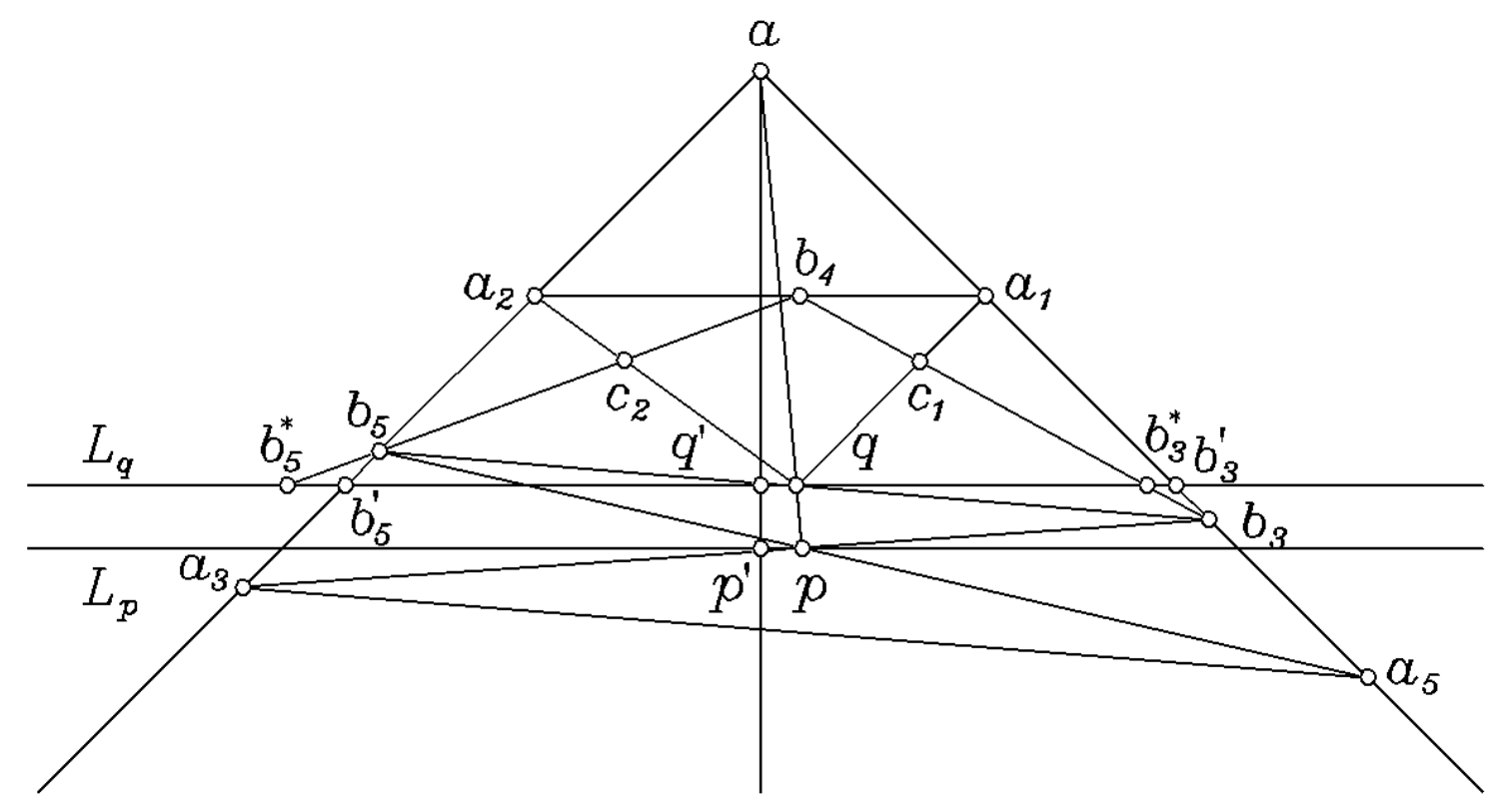

Figure 2

Take the intersection point $q$ of the segments $a p$ and $b_{3} b_{5}$. Denote the straight lines $y=p^{y}$ and $y=q^{y}$ by $L_{p}$ and $L_{q}$, respectively. Let $p^{\prime}$ and $q^{\prime}$ be the points $\left(0, p^{y}\right)$ and $\left(0, q^{y}\right)$, respectively, and let $h_{p^{\prime}}$ be the homothety with center $p^{\prime}$ and with homothety ratio $-\frac{1}{\nu(P, p)}$. Let us denote by $b_{3}^{\prime}$ and by $b_{5}^{\prime}$ the intersections of $L_{q}$ and of the straight lines $L\left(a, a_{1}\right)$ and $L\left(a, a_{2}\right)$, respectively. Let $a_{3}^{\prime}$ be the intersection of $L\left(a, a_{2}\right)$ and $L\left(p^{\prime}, b_{3}^{\prime}\right)$. Similarly, let $a_{5}^{\prime}$ be the intersection of $L\left(a, a_{1}\right)$ and $L\left(p^{\prime}, b_{5}^{\prime}\right)$.

We show that $b_{3}^{\prime}=h_{p^{\prime}}\left(a_{3}^{\prime}\right)$ and that $b_{5}^{\prime}=h_{p^{\prime}}\left(a_{5}^{\prime}\right)$. Observe that $p b_{3} b_{5}=h_{p}\left(p a_{3} a_{5}\right)$. Thus $a_{3} a_{5}$ and $b_{3} b_{5}$ are parallel and $b_{3} b_{5}$ is the homothetic image of $a_{3} a_{5}$ of ratio $\frac{1}{\nu(P, p)}$, where the center of homothety is $a$. Since $a_{3}^{\prime} a_{5}^{\prime}$ and $b_{3}^{\prime} b_{5}^{\prime}$ are also parallel, $b_{3}^{\prime} b_{5}^{\prime}$ is the homothetic image of $a_{3}^{\prime} a_{5}^{\prime}$ of ratio $\frac{1}{\nu(P, p)}$, where the center of homothety is $a$. Hence $\frac{\left|b_{3}^{\prime} b_{5}^{\prime}\right|}{\left|a_{3}^{\prime} a_{5}^{\prime}\right|}=\frac{1}{\nu(P, p)}$. From this we get that $b_{3}^{\prime} b_{5}^{\prime} p^{\prime}=h_{p^{\prime}}\left(a_{3}^{\prime} a_{5}^{\prime} p^{\prime}\right)$. That is, $b_{3}^{\prime}=h_{p^{\prime}}\left(a_{3}^{\prime}\right)$ and $b_{5}^{\prime}=h_{p^{\prime}}\left(a_{5}^{\prime}\right)$.

Denote $a_{1}$ by $a_{1}^{\prime}, a_{2}$ by $a_{2}^{\prime}, h_{p^{\prime}}\left(a_{1}\right)$ by $b_{1}^{\prime}$ and $h_{p^{\prime}}\left(a_{2}\right)$ by $b_{2}^{\prime}$. Let $a_{4}^{\prime}$ be the common point of the straight lines $L\left(a_{3}^{\prime}, b_{1}^{\prime}\right)$ and $L\left(a_{5}^{\prime}, b_{2}^{\prime}\right)$ and let $b_{4}^{\prime}$ denote $h_{p^{\prime}}\left(a_{4}^{\prime}\right)$. Using these notations we have $\frac{2\left|a_{i}^{\prime} p^{\prime}\right|}{\left|a_{i}^{\prime} b_{i}^{\prime}\right|}=\lambda(P, p)$ for $i=1, \ldots, 5$. We omit a consideration which shows that $P^{\prime}=a_{1}^{\prime} a_{2}^{\prime} a_{3}^{\prime} a_{4}^{\prime} a_{5}^{\prime}$ is a convex pentagon, that $p^{\prime}$ is in the kernel of $P^{\prime}$ and that $P^{\prime}$ has no 
side of $P^{\prime}$-length 2. From the above properties of $P^{\prime}$ and $p^{\prime}$ we get that $d_{P^{\prime}}\left(p^{\prime}, a_{i}^{\prime}\right)=\lambda(P, p)$ for $i=1,2,3,5$. We show that $d_{P^{\prime}}\left(p^{\prime}, a_{4}^{\prime}\right)>\lambda(P, p)$.

Take the points $c_{1}=h_{p}\left(b_{1}\right), c_{2}=h_{p}\left(b_{2}\right), c_{1}^{\prime}=h_{p^{\prime}}\left(b_{1}^{\prime}\right)$ and $c_{2}^{\prime}=h_{p^{\prime}}\left(b_{2}^{\prime}\right)$. As the homothety ratios of $h_{p}$ and $h_{p^{\prime}}$ are equal, we have $c_{1}^{y}=c_{2}^{y}=c_{1}^{\prime y}=c_{2}^{\prime y}$ and $\left|c_{1} c_{2}\right|=$ $\left|c_{1}^{\prime} c_{2}^{\prime}\right|$. Since $b_{3} b_{5}$ and $a_{3} a_{5}$ are parallel, the quadrangle $a_{5} b_{3} b_{5} a_{3}$ is a trapezoid. Thus $\left|b_{3} q\right|=\left|q b_{5}\right|$. Consider the triangles $b_{3} b_{3}^{\prime} q$ and $b_{5} b_{5}^{\prime} q$. We get that $\left|b_{3} b_{3}^{\prime}\right|=\left|b_{5} b_{5}^{\prime}\right|$. Let $b_{3}^{*}$ be the intersection point of the segment $b_{3} b_{4}$ and the straight line $L_{q}$. Similarly, let $b_{5}^{*}$ be the intersection point of the segment $b_{4} b_{5}$ and the straight line $L_{q}$. Notice that $b_{4}^{x}>0$, $b_{3} \in b_{3}^{\prime} a_{5}$ and that $b_{5} \in b_{5}^{\prime} a_{2}$. These observations and the fact that $\left|b_{3} b_{3}^{\prime}\right|=\left|b_{5} b_{5}^{\prime}\right|$ imply that $\left|b_{3}^{*} b_{5}^{*}\right|>\left|b_{3}^{\prime} b_{5}^{\prime}\right|$. Consider that $b_{4}$ is the intersection of $L\left(b_{3}^{*}, c_{1}\right)$ and $L\left(b_{5}^{*}, c_{2}\right)$ and that $b_{4}^{\prime}$ is the intersection of $L\left(b_{3}^{\prime}, c_{1}^{\prime}\right)$ and $L\left(b_{5}^{\prime}, c_{2}^{\prime}\right)$. As $\left|b_{3}^{*} b_{5}^{*}\right|>\left|b_{3}^{\prime} b_{5}^{\prime}\right|$ and $\left|c_{1} c_{2}\right|=\left|c_{1}^{\prime} c_{2}^{\prime}\right|$, we get that $b_{4}^{y}<b_{4}^{\prime}{ }^{y}$. Take the intersection point $b_{4}^{*}$ of $a_{1} a_{2}$ and $p^{\prime} b_{4}^{\prime}$. Since $\frac{\left|p^{\prime} a_{4}^{\prime}\right|}{\left|p^{\prime} b_{4}^{*}\right|}>\nu(P, p)$, we have $d_{P^{\prime}}\left(p^{\prime}, a_{4}^{\prime}\right)>\lambda(P, p)$. Obviously, $\lambda(P, p)=\lambda\left(P^{\prime}, p^{\prime}\right)$. Thus, according to Subcase 2.3 , the value $\lambda(P, p)$ cannot be maximal.

Notice that our choice of the side $a_{1} a_{2}$ was arbitrary. This implies that $\lambda(P, p)$ can be maximal only if $P$ is affine symmetric to every line containing the midpoint of a side of $P$ and the opposite vertex of $P$ and if $p$ is on every one of the above lines. But this holds only if $P$ is an affine regular pentagon and if $p$ is its center.

Lemma 2. Let $P=a_{1} a_{2} a_{3} a_{4} a_{5}$ be a convex pentagon and let $p$ be a point of $P$ which is not in the kernel of $P$. Then among $p, a_{1}, \ldots, a_{5}$ there exists a pair of points in $P$-distance less than $\lambda$.

Proof. If $P$ is a degenerate pentagon, then it has a chord containing at least 3 vertices of $P$. Thus in this case $P$ has a side of $P$-length at most 1 , which is less than $\lambda$.

In the sequel we deal with the case when $P$ is nondegenerate. Take a Cartesian coordinate system. As the $P$-distance of two points is affine invariant, we assume that the points $a_{1}, a_{2}$ and $a_{5}$ are $(0,0),(1,0)$ and $(0,1)$, respectively. Let $b$ be the point $(1,1)$. Denote the square $a_{1} a_{2} b a_{5}$ by $S$. Furthermore, for every $i, j \in\{1, \ldots, 5\}$ where $i \neq j$, we denote the slope of the line $L\left(a_{i}, a_{j}\right)$ by $m_{i j}$, provided it exists.

Case 1 , when $P$ has more than one side of $P$-length 2 . Consider the case when $P$ has two nonconsecutive sides of $P$-length 2 . We assume that $d_{P}\left(a_{1}, a_{2}\right)=2$ and that $d_{P}\left(a_{3}, a_{4}\right)=2$ (the proof of the other cases is analogous). Let the angle of $P$ at the vertex $a_{i}$ be denoted by $\alpha_{i}$ for $i=1, \ldots, 5$. From $d_{P}\left(a_{1}, a_{2}\right)=2$ we get that $\alpha_{1}+\alpha_{2} \leq \pi$. Similarly, from $d_{P}\left(a_{1}, a_{2}\right)=2$ we have $\alpha_{3}+\alpha_{4} \leq \pi$. The convexity of $P$ implies that $\alpha_{5} \leq \pi$. Obviously, $\sum_{i=1}^{5} \alpha_{i}=3 \pi$. Thus $\alpha_{1}+\alpha_{2}=\alpha_{3}+\alpha_{4}=\alpha_{5}=\pi$. Therefore $P$ is a degenerate pentagon.

Let us assume that $P$ has two consecutive sides of $P$-length 2 . Without loss of generality, let these sides be $a_{5} a_{1}$ and $a_{1} a_{2}$. Hence $P \subset S$. Denote the triangle $a_{1} a_{2} a_{5}$ by 
$S_{1}$. Take the homothetical copies $S_{2}, S_{3}, S_{4}$ of $a_{2} b a_{5}, S, a_{2} b a_{5}$ with ratio $\frac{1}{2}$ and with homothety centers $a_{2}, b, a_{5}$, respectively. As $P$ is convex, $S_{1}$ contains neither of the points $a_{3}$ and $a_{4}$ in its interior. If $S_{2}$ contains $a_{3}$ or if $S_{4}$ contains $a_{4}$, then $d_{P}\left(a_{2}, a_{3}\right) \leq 1$ or $d_{P}\left(a_{4}, a_{5}\right) \leq 1$, respectively. Finally, if $S_{3}$ contains both $a_{3}$ and $a_{4}$, then $m_{34}<0$ implies that $d_{P}\left(a_{3}, a_{4}\right) \leq 1$. Thus we get that $P$ has a side of $P$-length at most 1 .

Case 2, when $P$ has exactly one side of $P$-length 2 . We choose the indices of the points such that $d_{P}\left(a_{1}, a_{2}\right)=2$ and that $a_{3}^{y} \geq 1$. The condition of this case and the convexity of $P$ imply that $0<a_{4}^{x}<a_{3}^{x} \leq 1$ and that $1 \leq a_{3}^{y}<a_{4}^{y}$. Moreover, either $d_{P}\left(a_{1}, a_{5}\right)<\lambda$ or $a_{4}^{y} \leq \frac{1}{\tau}$. In the following we assume that $a_{4}^{y} \leq \frac{1}{\tau}<2$.

Observe that for arbitrary $w \in E^{2}$, the set of points whose $P$-distance from $w$ is less than $\lambda$ is the interior of the translate of $\frac{\tau}{2}(P-P)$ where the center of the body is $w$. From the previous considerations concerning the properties of $P$ we get that the sides of the centrally symmetric convex decagon $\frac{\tau}{2}(P-P)$ are parallel to $a_{1} a_{5}, a_{4} a_{5}, a_{1} a_{2}, a_{3} a_{4}, a_{2} a_{3}$.

First we show that if every side of $P$ has $P$-length greater than 1 , then $m_{45}>m_{13}$. We show the statement indirectly. Denote the intersection point of $L\left(a_{2}, a_{3}\right)$ and $L\left(a_{4}, a_{5}\right)$ by $s$ and denote the intersection point of $a_{1} a_{3}$ and $a_{2} a_{5}$ by $q$. Let $a_{3}^{\prime}$ and $a_{4}^{\prime}$ be the homothetic images of $a_{3}$ and $a_{4}$, respectively, where the center of homothety is $s$ and its ratio is 2 . Denote the midpoint of the segment $a_{5} s$ by $s_{5}$. Let $t$ be the point of $a_{2} a_{3}$ such that $a_{1} t$ and $a_{4} a_{5}$ are parallel. Similarly, let $s^{\prime}$ be the point of $L\left(a_{2}, a_{3}\right)$ such that $a_{5} s^{\prime}$ and $a_{1} a_{3}$ are parallel. Observe that $a_{1} t$ is a maximal chord of $P$ parallel to $a_{4} a_{5}$. Thus $d_{P}\left(a_{4}, a_{5}\right)>1$ implies that $\frac{1}{2}\left|a_{1} t\right|<\left|a_{4} a_{5}\right|$. Moreover, we have $\left|a_{5} s_{5}\right|=\frac{1}{2}\left|a_{5} s\right| \leq \frac{1}{2}\left|a_{1} t\right|$. Therefore $\left|a_{5} s_{5}\right|<\left|a_{4} a_{5}\right|$, from which $a_{4} \in s_{5} s$. Since $m_{13} \geq 1$, we get that $1 \leq \frac{\left|a_{2} q\right|}{\left|q a_{5}\right|}=\frac{\left|a_{2} a_{3}\right|}{\left|a_{3} s^{\prime}\right|}$. Hence $\frac{\left|a_{2} a_{3}\right|}{\left|a_{3} s\right|} \geq \frac{\left|a_{2} a_{3}\right|}{\left|a_{3} s^{\prime}\right|} \geq 1$. This and $a_{4} \in s_{5} s$ imply that $a_{3}^{\prime} a_{4}^{\prime}$ is a chord of $C$. Thus $d_{P}\left(a_{3}, a_{4}\right) \leq 1$, which is a contradiction.

Now we prove the statement under the condition that $m_{45}>m_{13}$. If $p$ is in both the triangles $a_{1} a_{3} a_{5}$ and $a_{2} a_{3} a_{5}$, then, according to the proof of Case 1 in Lemma 1 we have $d_{P}\left(a_{3}, p\right) \leq 1$ or $d_{P}\left(a_{5}, p\right) \leq 1$. We intend to examine the cases when $p \in a_{3} a_{4} a_{5}$, $p \in a_{1} a_{2} a_{5}$ or $p \in a_{2} a_{3} q$.

Subcase 2.1, when $p$ is in the triangle $a_{3} a_{4} a_{5}$. Denote the midpoints of the segments $a_{4} a_{5}, a_{3} a_{5}$ and $a_{3} a_{4}$ by $c_{3}, c_{4}$ and $c_{5}$, respectively. Notice that the triangles $a_{3} c_{4} c_{5}$, $a_{5} c_{3} c_{4}$ and the parallelogram $a_{4} c_{5} c_{4} c_{3}$ are contained in the homothetical copies of $P$ with homothety ratio $\frac{1}{2}$ where the homothety centers are $a_{3}, a_{5}$ and $a_{4}$, respectively. Thus in this case at least one of the values $d_{P}\left(a_{3}, p\right), d_{P}\left(a_{4}, p\right), d_{P}\left(a_{5}, p\right)$ is at most 1 .

Subcase 2.2, when $p$ is in the triangle $a_{1} a_{2} a_{5}$. We show the statement indirectly, therefore we assume that among $p$ and the vertices of $P$ there is no pair in $P$-distance less than $\lambda$. Denote by $Q_{1}$ and by $Q_{5}$ the translates of $\frac{\tau}{2}(P-P)$ where the centers of the bodies are $a_{1}$ and $a_{5}$, respectively. Consider the points $b_{1}=(1-\tau, 0)$ and $b_{5}=(1-\tau, 1-\tau)$. As $d_{P}\left(a_{2}, p\right) \geq \lambda$, we have $p \in a_{1} b_{1} b_{5} a_{5}$. We show that $a_{1} b_{1} b_{5} a_{5}$ is covered by the interiors of $Q_{1}$ and $Q_{5}$. For this it is enough to show that $b_{1} b_{5}$ is in the interior of $Q_{1} \cup Q_{5}$. Denote by $d_{1}$ the intersection of $b_{1} b_{5}$ and of the boundary of $Q_{1}$ such that $d_{1} \neq b_{1}$. Similarly, let $d_{5}$ 
be the intersection of $b_{1} b_{5}$ and of the boundary of $Q_{5}$. We omit an easy calculation that if $d_{1}$ is not on the side of $Q_{1}$ parallel to $a_{2} a_{3}$, or if $d_{5}$ is not on the side of $Q_{5}$ parallel to $a_{4} a_{5}$, then $b_{1} b_{5}$ is in the interior of $Q_{1} \cup Q_{5}$. In the opposite case we get that $d_{1}^{y}=m_{23}(1-2 \tau)$, and that $d_{5}^{y}=m_{45}(1-2 \tau)+1-\tau$. Thus $d_{1}^{y}-d_{5}^{y}=(2 \tau-1)\left(m_{45}-m_{23}\right)+\tau-1$.

Let us assume that $m_{34} \leq-1$. Take the point $u$ on the line $L\left(a_{2}, a_{3}\right)$ such that $a_{3}$ is the midpoint of the segment $a_{2} u$. As $d_{P}\left(a_{3}, a_{4}\right) \geq \lambda>1$, we have $\angle u a_{5} b<\angle a_{4} a_{5} b$. Thus, $0<\frac{2 a_{3}^{y}-1}{2 a_{3}^{x}-1}<m_{45}$. This implies that $d_{1}^{y}-d_{5}^{y} \geq(2 \tau-1)\left(\frac{2 a_{3}^{y}-1}{2 a_{3}^{x}-1}+\frac{a_{3}^{y}}{1-a_{3}^{x}}\right)+\tau-1 \geq$ $(2 \tau-1)\left(\frac{1}{2 a_{3}^{x}-1}+\frac{1}{1-a_{3}^{x}}\right)+\tau-1$. But the last expression is always positive.

Now we discuss the case when $m_{34}>-1$. In this case from $d_{P}\left(a_{3}, a_{4}\right) \geq \lambda$ we conclude that $a_{3}^{x}-a_{4}^{x} \geq \tau$. Consider the point $m=\left(0, \frac{1}{1-\tau}\right)$. Take the line $L_{m}$ through $m$ with slope -1 . Since $m_{34}>-1$ and since $d_{P}\left(a_{3}, a_{4}\right) \geq \lambda, a_{3}$ and $a_{4}$ are in the open halfplane not containing $a_{1}$ bounded by $L_{m}$. As $a_{4}^{y} \leq \frac{1}{\tau}$, we have $a_{4}^{x}>\frac{1}{1-\tau}-\frac{1}{\tau}$ and thus $a_{3}^{x}>\frac{1}{1-\tau}-\frac{1}{\tau}+\tau=\frac{11 \sqrt{5}-5}{20} \approx 0.980$. But this contradicts that $d_{1}$ is on the side of $Q_{1}$ parallel to $a_{2} a_{3}$, that is, that $a_{3}^{x} \leq \frac{1-\tau}{\tau}=\frac{\sqrt{5}+1}{4} \approx 0.809$. Hence $b_{1} b_{5}$ is in the interior of $Q_{1} \cup Q_{5}$. Therefore every point of $a_{1} b_{1} b_{5} a_{5}$ is in $P$-distance from $a_{1}$ or from $a_{5}$ less than $\lambda$.

Subcase 2.3, when $p$ is in the triangle $a_{2} a_{3} q$. Let $Q_{2}$ and $Q_{3}$ be the translates of $\frac{\tau}{2}(P-P)$ where the centers of the bodies are $a_{2}$ and $a_{3}$, respectively. If $p^{y}>1$, then $p$ is in the interior of $Q_{3}$. In the following we deal with the case when $p^{y} \leq 1$. From $d_{P}\left(a_{5}, p\right) \geq \lambda$ we have $p^{x} \geq \tau$. We show that the points of $a_{2} a_{3} q$ with $x$-coordinates at least $\tau$ are in the interior of $Q_{2} \cup Q_{3}$. Denote by $e_{2}$ the common point of the line $x=\tau$ and of the boundary of $Q_{2}$ with greater $y$-coordinate. Denote by $e_{3}$ the common point of the line $x=\tau$ and of the boundary of $Q_{3}$ with less $y$-coordinate. Let us show that $e_{2}^{y}-e_{3}^{y}$ is positive. We have $e_{3}^{y} \leq(1-\tau) a_{3}^{y}$. Moreover, $e_{2}^{y}=\tau a_{4}^{y}$ or $e_{2}^{y}=m_{45}(2 \tau-1)+\tau$. If $e_{2}^{y}=\tau a_{4}^{y}$, then $e_{2}^{y}-e_{3}^{y} \geq \tau\left(a_{3}^{y}+a_{4}^{y}\right)-a_{3}^{y}>0$. In the sequel we assume that $e_{2}^{y}=m_{45}(2 \tau-1)+\tau$. Observe that $m_{45} \geq m_{13} \geq 1$. Hence, if $a_{3}^{y}<\frac{3 \tau-1}{1-\tau}$, then $e_{2}^{y}-e_{3}^{y} \geq 3 \tau-1-(1-\tau) a_{3}^{y}>0$. Let us assume the opposite case, when $a_{3}^{y} \geq \frac{3 \tau-1}{1-\tau}$. In this case $a_{4}^{y} \leq \frac{1}{\tau}<a_{3}^{y}+\tau$. Thus $d_{P}\left(a_{3}, a_{4}\right) \geq \lambda$ implies that $a_{4}^{x} \leq a_{3}^{x}-\tau \leq 1-\tau$. Take the points $m\left(0, \frac{1}{1-\tau}\right)$ and $g\left(\frac{1}{\tau}, 1-\tau\right)$. Denote by $h$ the intersection point of $L(m, g)$ and $x=1$. We omit an elementary calculation which shows that $h^{y}=\frac{1}{\tau}-\frac{2 \tau-1}{(1-\tau)^{2}}$. Since $d_{P}\left(a_{3}, a_{4}\right) \geq \lambda$, we get that $a_{3}$ is in the closed halfplane containing $a_{1}$ bounded by $L\left(a_{4}, m\right)$. Therefore $a_{4}^{y}-a_{3}^{y} \geq \frac{1}{\tau}-h^{y}$. Thus, $a_{3}^{y} \leq a_{4}^{y}-\frac{1}{\tau}+h^{y} \leq h^{y} \approx 1.281$. But this contradicts our assumption that $a_{3}^{y} \geq \frac{3 \tau-1}{1-\tau} \approx 1.472$.

We have shown that $e_{2}^{y}-e_{3}^{y}$ is positive. But this implies that every point of the triangle $a_{2} a_{3} q$ with $x$-coordinate at least $\tau$ is in the interior of $Q_{2} \cup Q_{3}$.

Case 3, when $P$ has no side of $P$-length 2. We assume that $p$ is in the triangle $a_{1} a_{2} a_{5}$ and that $m_{34}$ is at least -1 (the proof of the other cases is analogous). Since $P$ has no side of $P$-length 2, we have $a_{3}^{x}>1$ and $a_{4}^{y}>1$. Observe that the points of $a_{1} a_{2} a_{5}$ with $x$-coordinate greater than $1-\tau$ are in $P$-distance from $a_{2}$ less than $\lambda$. Similarly, the points 
of $a_{1} a_{2} a_{5}$ with $y$-coordinate greater than $1-\tau$ are in $P$-distance from $a_{5}$ less than $\lambda$. Thus it is enough to deal with the case when both coordinates of $p$ are at most $1-\tau$. Take the point $f=\left(\frac{1}{\tau}-1, \frac{1}{\tau}-1\right)$. We intend to show that if $P$ has no side of $P$-length less than $\lambda$, then $f$ is in the interior of $P$.

Consider the case when the maximal chord parallel to $a_{4} a_{5}$ has an endpoint at $a_{1}$. In this case the other endpoint of the above chord is on the segment $a_{3} a_{4}$. This and $d_{P}\left(a_{4}, a_{5}\right) \geq \lambda$ imply that the $y$-coordinate of the common point of $L\left(a_{3}, a_{4}\right)$ and of the line $y=0$ is at least $\frac{1}{1-\tau}=\sqrt{5}$. Therefore, as $-1 \leq m_{34}$, we get that $f$ is in the open halfplane containing $a_{1}$ bounded by $L\left(a_{3}, a_{4}\right)$. Thus $f$ is in the interior of $P$.

Consider the case when the maximal chord of $P$ parallel to $a_{4} a_{5}$ has an endpoint at $a_{3}$. In this case $d_{P}\left(a_{4}, a_{5}\right) \geq \lambda$ implies that $a_{4}^{x} \geq \tau a_{3}^{x}$. Since $d_{P}\left(a_{3}, a_{4}\right) \geq \lambda$, we have $a_{3}^{x}-a_{4}^{x} \geq \tau$. Therefore $a_{4}^{x} \geq \frac{\tau^{2}}{1-\tau}$. From $d_{P}\left(a_{2}, a_{3}\right) \geq \lambda$ we get that $a_{3}$ is not in the interior of the homothetical copy of $a_{1} a_{2} a_{5}$ with homothety ratio $\tau$ where the image of $a_{1}$ is $a_{2}$. Take the points $a_{4}^{\prime}=\left(\frac{\tau^{2}}{1-\tau}, 1\right)$ and $a_{3}^{\prime}=(1,1-\tau)$. We omit an elementary calculation which shows that $f$ is in the open halfplane containing $a_{1}$ bounded by $L\left(a_{3}^{\prime}, a_{4}^{\prime}\right)$. Thus $f$ is in the open halfplane containing $a_{1}$ bounded by $L\left(a_{3}, a_{4}\right)$. Therefore $f$ is in the interior of $P$.

We have shown that if $P$ has no side of $P$-length less than $\lambda$, then $f$ is in the interior of $P$. But the definition of $f$ and our inequalities for the coordinates of $p$ imply that in this case $d_{P}\left(p, a_{1}\right)<\lambda$.

Lemma 3. Let $a_{1}, \ldots, a_{6}$ be points such that their convex hull $Q$ is a quadrangle or a triangle. Then among those points there exists a pair in $Q$-distance at most 1.

Proof. We show the statement of our lemma indirectly, we assume that among the points $a_{1}, \ldots, a_{6}$ there is no pair in $Q$-distance at most 1 . Let us take a Cartesian coordinate system. As the $Q$-distance of two points does not change under affine transformation, we assume that the points $a_{1}, a_{2}$ and $a_{3}$ are $(0,1),(0,0)$ and $(1,0)$, respectively. Take the point $b(1,1)$ and the square $S=a_{1} a_{2} a_{3} b$. We choose the indices of our points such that $Q \subset S$. Let us denote the homothetical copies of $S$ with homothety ratio $\frac{1}{2}$ and with centers $a_{1}, a_{2}, a_{3}, b$ by $S_{1}, S_{2}, S_{3}, S_{4}$, respectively. Consider the center $c$ of $S$, the center $b_{1}$ of the segment $a_{1} a_{2}$ and the center $b_{2}$ of the segment $a_{2} a_{3}$. Observe that every point of the triangle $a_{1} b_{1} c$ is in $Q$-distance at most 1 from $a_{1}$. Similarly, every point of the triangles $a_{2} b_{2} b_{1}$ and $b_{2} a_{3} c$ is in $Q$-distance at most 1 from $a_{2}$ and from $a_{3}$, respectively. Notice that there are no two points in the triangle $b_{1} b_{2} c$ in $Q$-distance from each other greater than 1. Thus $b_{1} b_{2} c$ contains at most one of the points $a_{4}, a_{5}, a_{6}$. Hence $Q$ is a quadrangle. Let $a_{4}$ be the fourth vertex of $Q$. As $d_{Q}\left(a_{1}, a_{4}\right)>1$ and $d_{Q}\left(a_{3}, a_{4}\right)>1$, we have $a_{4} \in S_{4}$. Hence every point of $S_{1}$ and $S_{3}$ is in $Q$-distance at most 1 from $a_{1}$ and $a_{3}$, respectively. Furthermore, $S_{4} \cap Q$ is covered by the homothetical copy of $Q$ with homothety center $a_{4}$ 
and with ratio $\frac{1}{2}$. Thus, every point of $S_{4} \cap Q$ is in $Q$-distance at most 1 from $a_{4}$. Moreover, $b_{2} c b_{1}$ contains at most one of the points $a_{5}$ and $a_{6}$, which is a contradiction.

Proof of Theorem. First, observe that if $C$ is an affine regular pentagon, and if the six points are its vertices and its center, then the minimal pairwise $C$-distance of the points is $\lambda$.

Take an arbitrary plane convex body $C$. Let $p_{1}, \ldots, p_{6}$ be points of $C$. Let us denote the convex hull of $p_{1}, \ldots, p_{6}$ by $C^{\prime}$. As $C^{\prime} \subset C$, the $C^{\prime}$-distance of arbitrary two points is greater than or equal to their $C$-distance. Theorem in [3] says that every convex hexagon has a side of relative length at most $8-4 \sqrt{3} \approx 1.072$. Thus, if $C^{\prime}$ is a hexagon, then among $p_{1}, \ldots, p_{6}$ there is a pair in $C^{\prime}$-distance at most $8-4 \sqrt{3}$, which is less than $\lambda$. With respect to Lemma 1 and Lemma 2, if $C^{\prime}$ is a pentagon, then the minimal pairwise $C^{\prime}$-distance of the points is at most $\lambda$, with equality if and only if $C^{\prime}$ is an affine regular pentagon and the points are its vertices and its center. According to Lemma 3 , if $C^{\prime}$ is a quadrangle or a triangle, then there exists a pair of points in $C^{\prime}$-distance at most 1 , which is less than $\lambda$. We have proved the first statement of our theorem.

To prove the second statement, it remains to show that if $C^{\prime}$ is an affine regular pentagon, the points are its vertices and its center and if there is no pair of them in a $C$-distance less than $\lambda$, then $C=C^{\prime}$. Let us choose the indices of the points such that $C^{\prime}$ is the pentagon $p_{1} p_{2} p_{3} p_{4} p_{5}$ and that $p_{6}$ is the center of $C^{\prime}$. Assume that $C \neq C^{\prime}$. In this case there exists a point $q \in C$, which is not a point of $C^{\prime}$ and the convex hull $D$ of $q$ and $C^{\prime}$ is a convex hexagon. It is enough to deal with the case when $D=p_{1} p_{2} p_{3} p_{4} p_{5} q$ (the proof of the other cases is analogous). But then $d_{C}\left(p_{6}, p_{3}\right) \leq d_{D}\left(p_{6}, p_{3}\right)<d_{C^{\prime}}\left(p_{6}, p_{3}\right)=\lambda$.

Finally, we conjecture that among seven arbitrary points of an arbitrary plane convex body there is a pair in relative distance at most 1 . The value 1 is attained, for instance, for the parallelogram. The example of the parallelogram shows that the estimate 1 is attained even for eight or nine points.

Corollary of [3] says that every convex heptagon has a side of relative length at most 1. From this and from Lemma 3 we immediately get that among eight or nine arbitrary points of an arbitrary plane convex body there is a pair in relative distance at most 1.

According to [2] or [4], we also conjecture that no plane convex body can be packed by its seven homothetical copies of ratio greater than $\frac{1}{3}$. Analogously, we observe that no plane convex body can be packed by its eight or nine homthetical copies of ratio greater than $\frac{1}{3}$.

\section{Appendix}

Statement. Among five arbitrary points of an arbitrary plane convex body there exists a pair in relative distance at most $\sqrt{5}-1$. 
Proof. Let $C$ be a plane convex body. Let $a_{1}, \ldots, a_{5}$ be points of $C$. Let us denote by $C^{\prime}$ the convex hull of $a_{1}, \ldots, a_{5}$. Observe that the $C^{\prime}$-distance of any two points is greater than or equal to their $C$-distance. Thus it is enough to show that among $a_{1}, \ldots, a_{5}$ there exists a pair in $C^{\prime}$-distance at most $\sqrt{5}-1$.

First, let us assume that $C^{\prime}$ is a triangle. Let us take the three segments whose endpoints are the midpoints of the sides of $C^{\prime}$. These segments divide $C^{\prime}$ into four homothetical copies of $C^{\prime}$, where the homothety ratio of three of them is $\frac{1}{2}$ and the homothety

ratio of the remaining one is $-\frac{1}{2}$. Observe that none of the above homothetical copies contains two points in $C^{\prime}$-distance greater than 1 . Moreover, there is a copy containing at least two of the points $a_{1}, \ldots, a_{5}$. Hence, among the points $a_{1}, \ldots, a_{5}$ there is a pair in $C^{\prime}$-distance at most 1 , which is less than $\sqrt{5}-1$.

Second, let us assume that $C^{\prime}$ is a quadrangle. Observe that there exists a parallelogram $P$ containing $C^{\prime}$ such that two its consecutive sides are sides of $C^{\prime}$. Without loss of generality, let $C^{\prime}=a_{1} a_{2} a_{3} a_{4}$ and let $a_{1}, a_{2}, a_{3}$ be vertices of $P$. Denote the fourth vertex of $P$ by $b$.

If $a_{5}$ is a boundary point of $C^{\prime}$, then the $C^{\prime}$-distance of $a_{5}$ and a vertex of $C^{\prime}$ is at most 1. Thus it is enough to deal with the case when $a_{5}$ is in the interior of $C^{\prime}$.

In the following, let us assume indirectly that the pairwise $C^{\prime}$-distances of $a_{1}, a_{2}, a_{3}$, $a_{4}$ and $a_{5}$ are greater than $\sqrt{5}-1$. Let $P_{1}$ and $P_{3}$ be the homothetic images of $P$ with homothety ratio $\frac{1}{2}(\sqrt{5}-1)$, where the centers of homothety are $a_{1}$ and $a_{3}$, respectively. Let $P_{2}$ and $P_{4}$ be the homothetic images of $P$ with homothety ratio $\frac{1}{2}(3-\sqrt{5})$, where the centers of homothety are $a_{2}$ and $b$, respectively. Hence $P=P_{1} \cup P_{2} \cup P_{3} \cup P_{4}$.

Notice that the $C^{\prime}$-distance of every point of $P_{1}$ and $P_{3}$ is at most $\sqrt{5}-1$ from $a_{1}$ and from $a_{3}$, respectively. Hence $a_{4}$ and $a_{5}$ are in $P_{2}$ or $P_{4}$. As $C^{\prime}$ is convex, $a_{4} \notin P_{2}$ and therefore it is in $P_{4}$. Observe that the $C^{\prime}$-distance of every point of $P_{4} \cap Q$ is less than $\sqrt{5}-1$ from $a_{4}$. Thus $a_{5}$ is in $P_{1}$.

Let $u=P_{1} \cap P_{3} \cap P_{4}$ and let $v=P_{1} \cap P_{2} \cap P_{3}$. Notice that $v$ is the homothetic image of $u$, where the center of homothety is $a_{1}$ and the homothety ratio is $\frac{1}{2}(\sqrt{5}-1)$. Therefore $P_{1}$ is contained in the homothetic image of $C^{\prime}$, where the center of the homothety is $a_{1}$ and the homothety ratio is $\frac{1}{2}(\sqrt{5}-1)$. From this we have $d_{C^{\prime}}\left(a_{1}, a_{5}\right) \leq \sqrt{5}-1$, which contradicts our indirect assumption.

The proof of the third case, when $C^{\prime}$ is a convex pentagon, is given in a detailed form in [1].

\section{References}

[1] K. Doliwka, M. Lassak, On relatively short and long sides of convex pentagons, Geom. Dedicata 56 (1995), 221-224.

[2] P. G. Doyle, J. C. Lagarias, D. Randall, Self-packing of centrally symmetric convex bodies in $R^{2}$, Discrete Comput. Geom. 8 (1992), 171-189. 
[3] Z. Lángi, On the relative lengths of sides of convex polygons, Studia Sci. Math. Hungar. 40 (2003), 115-120.

[4] Z. Lángi, M. Lassak, Relative distance and packing a body by homothetical copies, Geombinatorics 13 No. 1 (2003), 184-190.

[5] M. Lassak, On five points in a plane convex body in at least unit relative distances, Coll. Math. Soc. János Bolyai 63 Intuitive Geometry, Szeged (1991), 245-247.

KÁROLY BÖRÖCZKY

Eötvös Loránd University, Department of Geometry

1117 Budapest, PÁzmány P. SÉtány 1/C, Hungary

E-mail: boroczky@cs.elte.hu

ZsOLT LÁNGI

College of Dunaújváros, Institute of Natural Sciences

2400 Dunaújváros, Táncsics M. Út 1/A, Hungary

E-mail: zslangi@kac.poliod.hu 